\title{
Load Planning of Transport Aircraft Based on Hybrid Genetic Algorithm
}

\author{
Yang Chenguang ${ }^{1, a}$, Liu $\mathrm{Hu}^{1}$ and Gao Yuan ${ }^{2}$ \\ ${ }^{1}$ School of Aeronautical Science and Engineering, Beihang University, Beijing, 100191, China \\ ${ }^{2}$ Power Electronics, Machines and Control Research Group, Faculty of Engineering, University of Nottingham, \\ Nottingham, NG7 2RD, UK
}

\begin{abstract}
Loading of transport aircraft attracts much attention as the airlift is developing rapidly. It refers to the process that various cargoes are loaded, in an appropriate manner, into kinds of transport aircrafts with constraints of volume, weight and gravity center. Based on two-dimensional bin packing with genetic algorithm (GA), a new hybrid algorithm is proposed to solve the multi-constraint loading problem of transport aircraft for seeking the minimum of fuel consumption. Heuristic algorithm is applied to optimize single-aircraft loading in GA decoding, and the procedure of hybrid GA is summarized for the multi-aircraft loading issues. In the case study, eight kinds of cargos are distributed in three different aircrafts. The optimal result indicates that this algorithm can rapidly generate the best plan for the loading problem regarding lower transport costs.
\end{abstract}

\section{Introduction}

Airlift is a transportation way with good properties like speed, safety and high-capacity. It has become one of the most important means of transportation especially for large and urgent cargoes. Similar to other planning researches, the motivations of studying transport aircraft loading here include the premier goal to reduce the cost of transportation. The former study of transport aircraft loading is mainly focused on the single aircraft loading [1] [2] [3]. In addition, the method for multi-aircraft cargo loading under different constraints is still primitive [4] [5]. Algorithms like "Best Fit Level Decreasing" (BFLD) [1], "Command Line Sequencing" (CLS) [2] etc. are usually utilized for transport aircraft loading. Vancroonenburg W [3] introduces a mixed integer linear programming model for aircraft load planning with multi-constraint. However, those methods are limited to the loading of single-aircraft freight. Roesener [4] developed a tabu search based heuristic to solve a multi-aircraft problem which accounts for aircraft specific constraints. However, the authors just focused on loading pallets for only one kind of cargo. Although Liu [5] proposed an algorithm that can automatically generate cargo loading plans for the transport aircraft fleet, the number of plans is far from generating an optimized airlift plan.

With heuristic algorithm (HA) utilized for single-aircraft loading, a new hybrid genetic algorithm (HGA) is proposed to solve multi-constraint loading problem of transport aircraft for minimizing the

\footnotetext{
${ }^{a}$ Corresponding author : transport_plane@163.com
} 
fuel consumption. This algorithm can rapidly generate the optimized plans for transport aircraft loading.

\section{Loading problem of transport aircraft}

The problem of transport aircraft loading can be described as follows: With given cargoes and a fleet of transport aircrafts, how to reasonably arrange the cargo position on each aircraft under the constraints of volume, weight and center of gravity. The cost of transportation (referring to fuel consumption here) could be reduced by using some optimization methods. Transport aircraft loading is the design and optimization of two problems. One is for the loading position of cargos on single aircraft, and the other is to generate the loading order of transport aircrafts.

According to the characteristics and requirements of transport aircraft loading, design principles and simplifications can be given as follows:

- Since cargo is generally not stacked inside of the aircraft. Transport aircraft loading can be simplified as a two-dimensional packing problem [6].

- The shape of cargo hold of transport aircraft is simplified as a rectangular.

- The shape of cargo is simplified as a rectangular, and its center of gravity is its geometric center.

The optimization of aircraft loading is a Non-deterministic Polynomial (NP) complete problem [7]. Most of new GA methods have been challenged by the increasing complexity of formulation and computation; therefore, HGA is preferable because of its practicability for packing freight into a cargo hold.

On the one hand, with multiple constraints of space planning, it is not that easy to obtain optimal solution by exact algorithms. Therefore, HA is utilized to solve this problem. On the other hand, Genetic algorithm (GA) is a general and effective method to solve the loading order problem of transport aircrafts. HGA, which combines GA with a heuristic rule, is designed in this study for aircraft loading problem. Its basic thinking is that: decoding chromosome order using heuristic rule before fitness calculation; and then, an optimal solution (loading order of transport aircraft) is produced using GA.

\section{Design of HA for single-aircraft loading}

Single-aircraft loading can be simplified as a two-dimensional (top-view) packing problem. A singleaircraft loading algorithm has been proposed by Liu [5], this study improves it using heuristic algorithm. For each cargo to be loaded, there are two basic requirements that should be met: its height must be less than the height of the cargo hold; its total weight must be less than the remaining loading capacity of the transport aircraft.

It is noting that, regarding any part of the cabin hold for loading cargos, the loading priority order is sorted from maximum to minimum by their side lengths. The loading procedure is concluded as follows:

1) Rank all cargos by their lengths (not widths) and choose an aircraft randomly.

2) A virtual cabin (VC) needs to be constructed for the first cargo (which can be loaded firstly following the length order), and it should be positioned at the left bottom of cargo hold.

3) Then, the following cargoes should be loaded in the right space of this VC. For this space, try all cargos one by one following the length order until one is available. After loading the second cargo, if there still be enough space left in the right of this VC, try all cargos in the same way until the smallest cargo cannot be loaded on the right.

4) Consider the space of top right corner as a sub VC, and compare its two side lengths: 1 . if the vertical length is larger than the horizontal length, load the cargos following the length order in the sub VC from bottom to top (follow step 3 in horizontal direction); 2. if the vertical is smaller than the horizontal, all cargos left should be rotated 90 degrees, and they will be loaded following the same length order in the sub $\mathrm{VC}$ from right to left (if needed, add the second cargo level upward in this sub VC). 
5) If some cargos still fail to be loaded after Step 4, a new VC needs to be built. They can be loaded following step 3-4.

6) If there is no enough space to contain another new VC which means all vertical side lengths of the left cargos are larger than the remaining vertical length in this aircraft, another transport aircraft should be utilized for the cargos.

As shown in Figure 1, cargos, including 1 A, 1 B, 3 C, 2 D, 2 E, are ready to be loaded in one aircraft. According to above rules, $\mathrm{VC} 1$ should be firstly constructed and Cargo A is loaded in the left bottom of this $\mathrm{VC}$. Then load Cargo $\mathrm{C}$ on the right of $\mathrm{A}$, followed by considering the top right corner of $\mathrm{VCl}$ as a sub VC. Its vertical length is larger than the horizontal, the other Cargo $\mathrm{C}$ is loaded vertically and one Cargo E is loaded on the top of C based on the rules in Step 4. Finally, VC2 is built for loading remaining B, C, 2 D and E. Different from VC1, all left cargos need to be rotated 90 degree before loading in its sub $\mathrm{VC}$, and then $1 \mathrm{D}, 1 \mathrm{E}$ are loaded from right to left there.

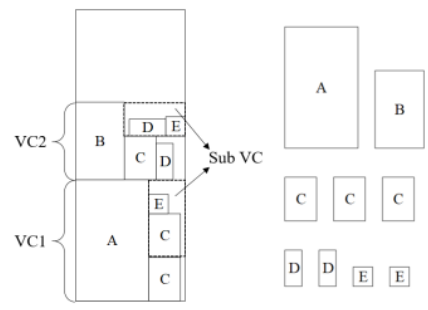

Figure 1. The HA of the single-aircraft loading.

The centers of gravity for all the cargos on the same aircraft should be arranged within a tolerance range for safety purpose. To satisfy the longitudinal balancing requirement, a method called "Pyramid Loading" [5] is utilized. During this balancing process, the cargos in one VC should be considered as a whole, their relative positions will not be changed.

For the vertical balance, firstly rank all VCs from heavy to light and they can be represented by VC1, VC2, VC3 etc. Then reload all VCs. Locate the heaviest one (VC1) in the middle of cargo hold. Put the second-heaviest (VC2) on the bottom of VC1 followed by putting VC3 on the top of VC1. Then VC4 is putted on the bottom of VC2, VC5 on the top of VC3. By that analogy, all VCs can be vertically positioned. If longitudinal balance is not met or any VC is out of the cargo hold, all VCs should be combined into one bigger $\mathrm{VC}$ and move it to a suitable position in the cargo hold.

For the horizontal balance, firstly calculate the rolling moment of all VCs, and rank the absolute values from large to small. Each VC need to be confirmed one by one, whether it should be symmetrically reversed from left to right, by the following three aspects: 1 . rolling moment; 2 . the sum of rolling moments of former VCs; 3 . this VC contains only one kind of cargo or not. The procedure is shown in Figure 2.

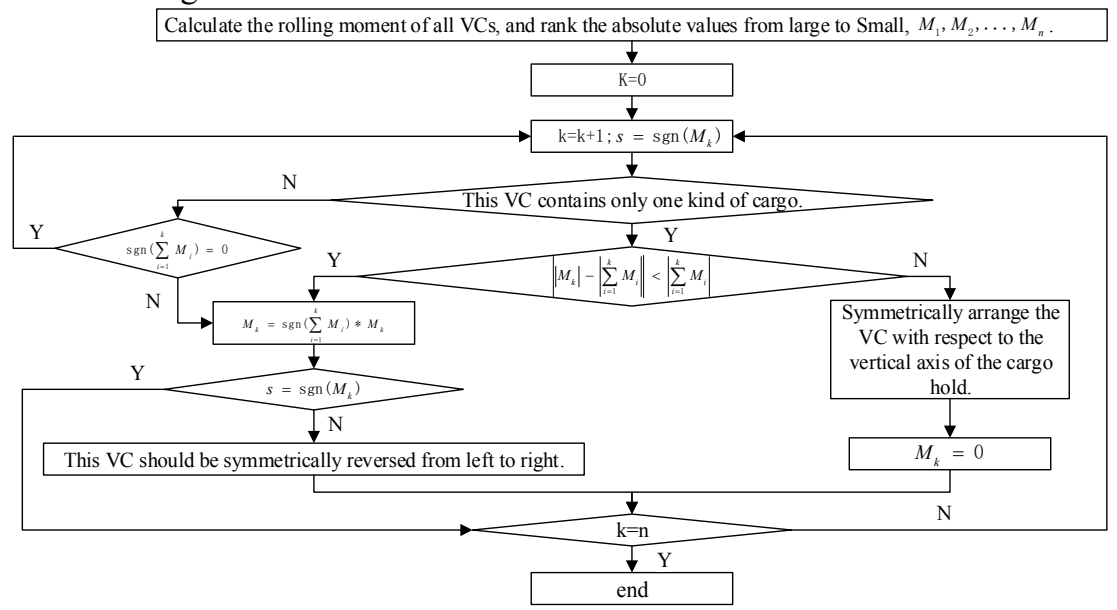

Figure 2. Horizontal balance procedure. 


\section{Design of HGA for multi-aircraft loading}

HGA is used to solve the loading problem with more than one transport aircraft. The procedure of GA based on the heuristic algorithm is shown in Figure 3.

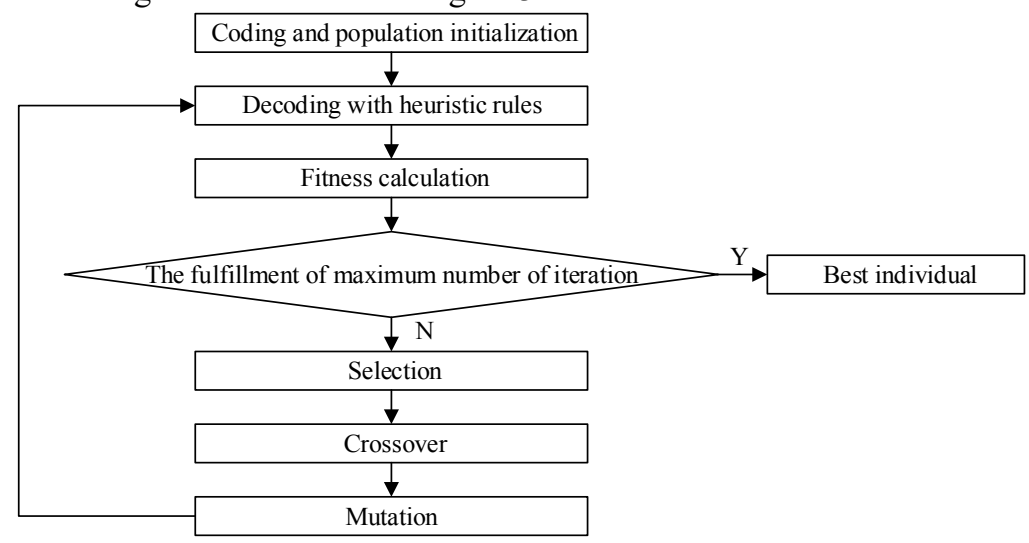

Figure 3. The basic procedures of HGA.

(1) Coding

Serial number coding is employed according to the characteristics of the loading. All the transport aircrafts are randomly arranged in a line, forming a chromosome. Their individual lengths equal to $L=\sum_{i=1}^{k} n_{\mathrm{i}}$. For example, three types of aircraft (marked by A, B, C respectively, $\mathrm{k}=3$ ), and the numbers of each type of aircraft are $3,4,2$. One individual serial number generated might be BCBAACBAB (including three "A", four "B" and two " $C$ "), and the individual length is $L=9$.

(2) Population initialization

Assume that the scale of GA population in the execution is $\mathrm{N}$. Repeat the individual coding for $\mathrm{N}$ times, individuals can be generated $x_{i}(i=1,2, \ldots, N)$, and the initial population $X=\left\{\mathrm{x}_{1}, x_{2}, \ldots, x_{N}\right)$, whose scale is $\mathrm{N}^{*} \mathrm{~L}$, is finally confirmed.

(3) Decoding with heuristic rules

Based on the heuristic algorithm, all the cargos are loaded following a chromosome order of transport aircraft until the loading mission is complete. The total loading weight of each transport aircraft $m_{j}$ should be recorded.

(4) Fitness calculation

The operational costs of the flights are calculated by empirical data under the flight mission profile. The fuel consumption of each loaded transport aircraft, $F_{j}\left(m_{j}\right)(j=1,2, \ldots, P)$, is a function of loading weight and it can be calculated by interpolation method based on the fuel consumption of some typical loading weights. According to loading plans mentioned above, the total fuel consumption of the cargo aircraft is $F=\sum_{j=1}^{P} F_{j}\left(M_{j}\right)$. The fitness value $f\left(x_{i}\right)$ of $x_{i}$ individual can be recorded as the reciprocal of $\left(F-c_{\min }\right) . c_{\min }$ is the lowest estimated magnitude of fuel consumption under current loading information.

(5) Genetic operation

Selection: roulette selection based on the elitist strategy.

Crossover: single-point crossover is applied, and the crossover probability is set as $p_{c}$; the crossover individuals $x_{i}, x_{j}$ are randomly selected according to $p_{c}$; the crossover point in chromosome is randomly selected. 
Mutation: randomly select the mutation position, and then change the generic code; the mutation probability is set as $p_{m}$. (The inversion operation can be added after mutation in order to improve the ability of local exploration.)

Termination condition: the maximum number of iteration.

\section{Case study}

The transport aircrafts in $A$ airport are three Y-9, four IL-76 and two C-17 (Because the data of typical large transport aircraft Y-20 is still unknown, replace $\mathrm{Y}-20$ with $\mathrm{C}-17)$. The parameters of the cargo to be transported are displayed in Table 1. The distance between A and B is 1300 kilometers. The optimal loading plan of the aircrafts transporting these cargos from A to B is to be solved for the minimum target of fuel consumption.

Table 1. Cargo parameters.

\begin{tabular}{cccccc}
\hline Cargo & Length $(\mathrm{m})$ & Width $(\mathrm{m})$ & Height $(\mathrm{m})$ & Weight $(\mathrm{t})$ & Number \\
\hline Command vehicle & 7.000 & 3.000 & 2.900 & 14.600 & 3 \\
Communication vehicle & 6.300 & 2.500 & 2.200 & 15.200 & 2 \\
Ambulance & 6.000 & 2.300 & 2.300 & 6.000 & 3 \\
IC container & 6.058 & 2.438 & 2.438 & 15.000 & 3 \\
JY10 container & 4.012 & 2.438 & 2.438 & 5.000 & 4 \\
JY7 container & 2.438 & 1.968 & 2.100 & 3.500 & 3 \\
JY5 container & 2.438 & 1.457 & 2.100 & 1.500 & 7 \\
JY1 container & 1.300 & 0.900 & 1.300 & 0.800 & 7 \\
\hline
\end{tabular}

In this case study, HGA is developed in C language. For the initial parameters of GA, scale of population $\mathrm{N}=10$, maximum number of iteration $\mathrm{T}=200$, crossover probability $p_{c}=0.6$ and mutation probability $p_{m}=0.1$.

Optimal results using HGA is shown in Figure 4. The first point of convergence happens at the 73th generation which has a minimum cost value of 184.35. The final result of the optimized loading order is C-17, Y-9, C-17, IL-76, and the loading sequence of each cargo is monotonically decreasing regarding their lengths. The total fuel consumption is $184.35 \mathrm{t}$ in this optimal plan. The optimal loading of each transport aircraft is shown in Figure 5. As the initial fuel consumption is $243.89 \mathrm{t}$, transport cost is reduced greatly by using HGA.

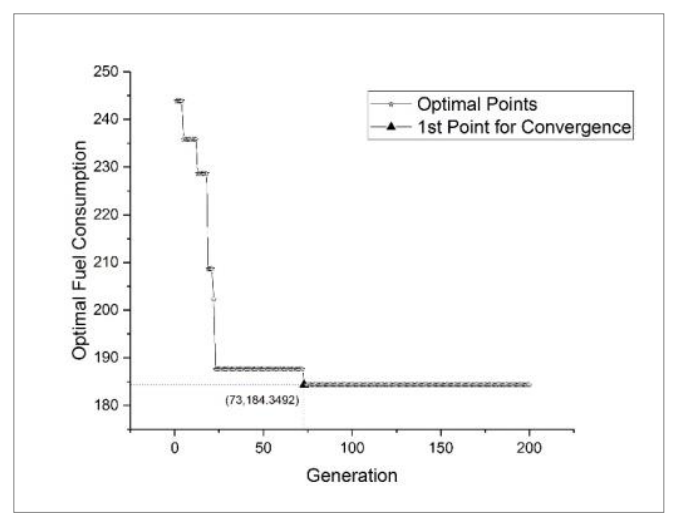

Figure 4. Optimal results using HGA. 

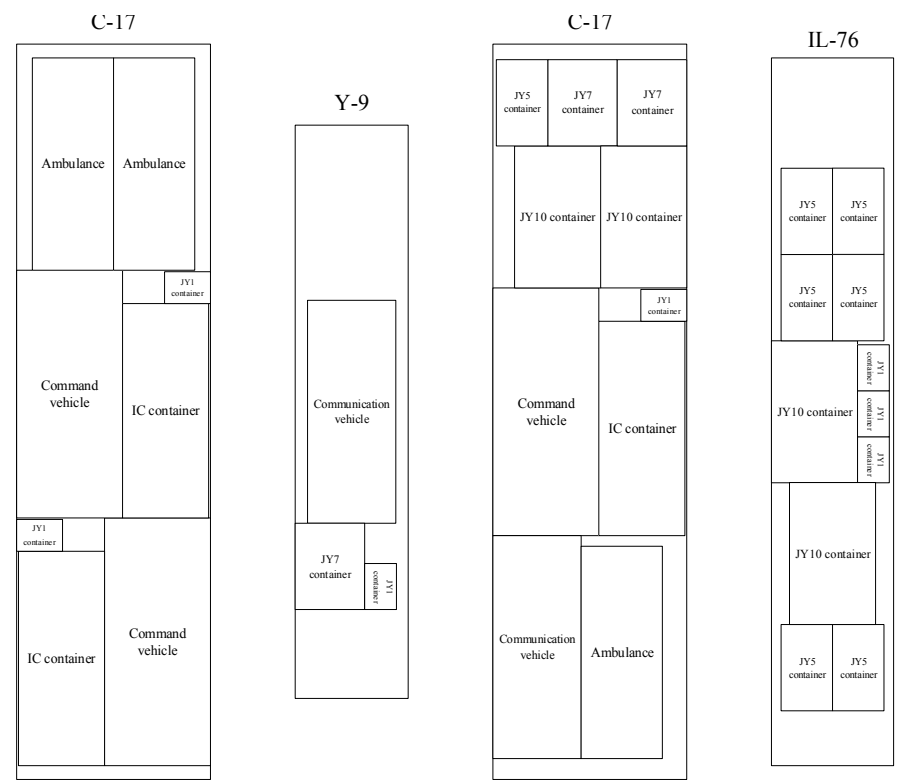

Figure 5. The optimal loading of each transport aircraft.

\section{Conclusion}

Considering various constraints of airlift loading, a new HGA is proposed for the aircraft loading problem. The interest of this algorithm is to combine HA of single-aircraft loading with GA based on two-dimensional bin planning, making easier the convergence of loops. The high efficiency and feasibility of HGA are proved in the case study. The optimal loading plan generated lowers the total fuel consumption and improves the efficiency of transportation, offering better auxiliary options for the load planning of transport aircraft.

\section{References}

1. Gehring H, Menschner K, Meyer M 1990 A computer-based heuristic for packing pooled shipment containers. European Journal of Operational Research, vol 44, no 2, pp 277-288.

2. Heidelberg, Kurt R 1995 A Bin Packing Algorithm for Cargo Conveyance Systems. IN Virginia Commonwealth University.

3. Vancroonenburg W, Verstichel J, Tavernier K, Berghe G V 2014 Automatic air cargo selection and weight balancing: a mixed integer programming approach. Transportation Research Part E Logistics \& Transportation Review, vol 65, no 2, pp 70-83.

4. Roesener, August G 2006 An Advanced Tabu Search Approach to the Airlift Loading Problem. IN The University of Texas at Austin.

5. Liu Ningjun, Wang Lixin, Pan Wenjun 2013 An Optimal Method of Transport Aircraft Fleet Cargo Loading. Journal of Beijing University of Aeronautics and Astronautics, vol 39, no 6, pp 751-755.

6. Kurt R, Heidelberg, Gregory S, Parnell, James E, Ames IV 1998 Automated air load planning. Naval Research Logistics, vol 45, no 8, pp 751-768.

7. Garey M R, Johnson D S 1979 Computers and Intractability: A Guide to the Theory of NPCompleteness. W. H. Freeman Press, p 113. 